\title{
OPEN Practical approach to detection and surveillance of emerging highly resistant Mycobacterium tuberculosis Beijing 1071-32-cluster
}

Igor Mokrousov ${ }^{1 \bowtie}$, Anna Vyazovaya ${ }^{1}$, Viacheslav Sinkov ${ }^{2}$, Alena Gerasimova ${ }^{1}$, Panayotis loannidis ${ }^{3}$, Weiwei Jiao ${ }^{4}$, Polina Khromova ${ }^{1,2}$, Dimitrios Papaventsis ${ }^{3}$, Oksana Pasechnik ${ }^{5}$, João Perdigão ${ }^{6}$, Nalin Rastogi ${ }^{7}$, Adong Shen ${ }^{4,8}$, Yuriy Skiba ${ }^{9}$, Natalia Solovieva ${ }^{10}$, Philip Suffys ${ }^{11}$, Silva Tafaj ${ }^{12}$, Tatiana Umpeleva ${ }^{1,13}$, Diana Vakhrusheva ${ }^{13}$, Irina Yarusova ${ }^{1,14}$, Svetlana Zhdanova ${ }^{2}$, Viacheslav Zhuravlev ${ }^{10}$ \& Oleg Ogarkov ${ }^{2}$

Ancient sublineage of the Mycobacterium tuberculosis Beijing genotype is endemic and prevalent in East Asia and rare in other world regions. While these strains are mainly drug susceptible, we recently identified a novel clonal group Beijing 1071-32 within this sublineage emerging in Siberia, Russia and present in other Russian regions. This cluster included only multi/extensive drug resistant (MDR/XDR) isolates. Based on the phylogenetic analysis of the available WGS data, we identified three synonymous SNPs in the genes Rv0144, Rv0373c, and Rv0334 that were specific for the Beijing 1071-32-cluster and developed a real-time PCR assay for their detection. Analysis of the 2375 genetically diverse $M$. tuberculosis isolates collected between 1996 and 2020 in different locations (European and Asian parts of Russia, former Soviet Union countries, Albania, Greece, China, Vietnam, Japan and Brazil), confirmed $100 \%$ specificity and sensitivity of this real-time PCR assay. Moreover, the epidemiological importance of this strain and the newly developed screening assay is further stressed by the fact that all identified Beijing 1071-32 isolates were found to exhibit MDR genotypic profiles with concomitant resistance to additional first-line drugs due to a characteristic signature of six mutations in rpoB450, rpoC485, katG315, katG335, rpsL43 and embB497. In conclusion, this study provides a set of three concordant SNPs for the detection and screening of Beijing 1071-32 isolates along with a validated real-time PCR assay easily deployable across multiple settings for the epidemiological tracking of this significant MDR cluster.

\author{
Abbreviations \\ MDR Multidrug resistance \\ XDR Extensive drug resistance \\ WGS Whole genome sequencing
}

\begin{abstract}
${ }^{1}$ St. Petersburg Pasteur Institute, 14 Mira Street, St. Petersburg, Russia 197101. ${ }^{2}$ Scientific Centre of the Family Health and Human Reproduction Problems, Irkutsk, Russia. ${ }^{3}$ National Reference Laboratory for Mycobacteria, Sotiria Chest Diseases Hospital, Athens, Greece. ${ }^{4}$ National Clinical Research Center for Respiratory Diseases, Beijing Key Laboratory of Pediatric Respiratory Infection Disease, Beijing Pediatric Research Institute, Beijing Children's Hospital, Capital Medical University, National Center for Children's Health, Beijing, China. ${ }^{5}$ Omsk State Medical University, Omsk, Russia. ${ }^{6}$ Research Institute for Medicines (iMed.ULisboa), Faculdade de Farmácia, Universidade de Lisboa, Lisbon, Portugal. ${ }^{7}$ WHO Supranational TB Reference Laboratory, Unité de la Tuberculose et des Mycobactéries, Institut Pasteur de la Guadeloupe, Abymes, Guadeloupe, France. ${ }^{8}$ Children's Hospital Affiliated to Zhengzhou University, Henan Children's Hospital, Zhengzhou Children's Hospital, Zhengzhou, China. ${ }^{9}$ Almaty Branch of National Center for Biotechnology in Central Reference Laboratory, Almaty, Kazakhstan. ${ }^{10}$ St. Petersburg Research Institute of Phthisiopulmonology, St. Petersburg, Russia. ${ }^{11}$ Fundação Oswaldo Cruz, Rio de Janeiro, Brazil. ${ }^{12}$ University Hospital "Shefqet Ndroqi", Tirana, Albania. ${ }^{13}$ Ural Research Institute for Phthisiopulmonology, National Medical Research Center of Tuberculosis and Infectious Diseases of Ministry of Health of the Russian Federation, Yekaterinburg, Russia. ${ }^{14} \mathrm{Clinical}$ Tuberculosis Dispensary, Omsk, Russia.『email: igormokrousov@yahoo.com
\end{abstract}




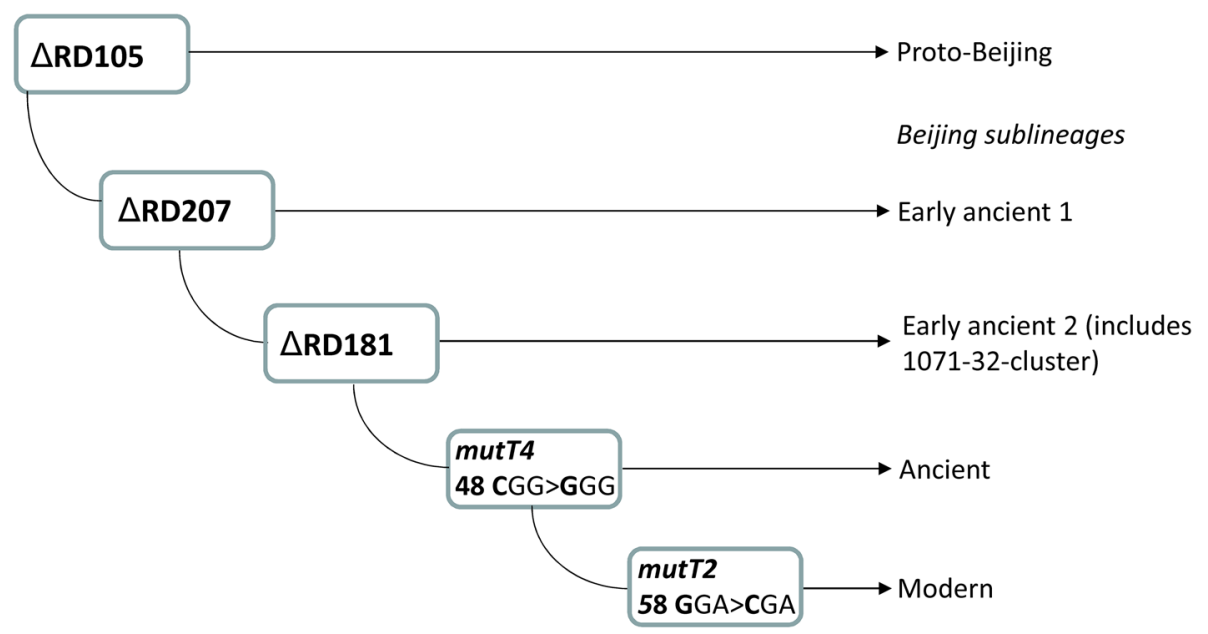

Figure 1. Simplified evolutionary pathway of the M. tuberculosis Beijing genotype. Digital profile of the 1071-32-cluster: 244231342644425173353923 (loci are listed in clockwise order on chromosome).

NGS Next generation sequencing

SNP Single nucleotide polymorphism

MIRU Mycobacterial interspersed repetitive units

VNTR Variable number of tandem repeats

Mycobacterium tuberculosis was initially regarded as a highly homogeneous population, yet, recent data demonstrated that this species is more genetically and functionally diverse than previously appreciated with diverging clinical significance associated with some of its phylogenetic clades, genetic families, or clonal complexes ${ }^{1}$. Studies carried out in the previously unexplored geographical regions and reanalysis of the available M. tuberculosis strains were able to pinpoint clinically and/or epidemiologically significant genotypes emerging in different world regions. In-depth analysis of such genotypes allows for detection of their specific markers associated with pathobiologically significant features such as drug resistance, hypervirulence, or transmissibility.

The Beijing genotype remains the most studied genetic family of $M$. tuberculosis. It can be robustly subdivided into phylogenetically ancient/ancestral and modern sublineages. Initially, subdivision was based on IS6110-RFLP typing, with ancient strains having classical spoligotypes and atypical IS6110-RFLP profiles ${ }^{2}$. Further studies had identified additional markers, mostly SNP and large sequence polymorphisms, that allowed some degree of discrimination within the ancient sublineage ${ }^{3,4}$ (Fig. 1). To date, WGS and high-resolution MIRU-VNTR analysis have proven useful for the identification of epidemic clusters but mostly within the globally dispersed modern Beijing sublineage ${ }^{5-9}$. In contrast, strains of the ancient Beijing sublineage circulate mainly in East Asia (K-strain in Korea is a known example ${ }^{10}$ ) and are rarely isolated elsewhere. Consequently, these strains are less studied, their epidemic impact is underestimated and their emergence in locations beyond East Asia is unexpected and needs further attention.

Modern Beijing strains are dominant in the former Soviet Union, the most notorious clusters being the Russian epidemic strain B0/W $148^{8}$ and Central Asia Outbreak clade ${ }^{9}$. Both genotypes have been described in other countries and in some studies they were shown to circulate outside immigrant communities, highlighting their potential to spread to the autochthonous population ${ }^{11-13}$. Ancient Beijing strains in Russia were much less studied because they accounted for only $2 \%$ of the local $M$. tuberculosis populations ${ }^{2}$. Interestingly, a recent study in the Omsk province in Western Siberia detected a relatively elevated $10 \%$ prevalence of the ancient Beijing sublineage ${ }^{14}$. Most of those isolates belonged to the 1071-32-cluster (according to the MIRU-VNTRplus. org MLVA MtbC15-9 classification scheme) and also formed a monophyletic cluster within the WGS-based phylogenetic tree ${ }^{15}$. All Russian isolates of this cluster (from St. Petersburg, Omsk, and Samara) were multidrug resistant (MDR) due to the specific signature of six mutations in $r p o B, r p o C, k a t G, r p s L$ and embB. It was complemented in most isolates with additional mutations in $p n c A, g y r A$, rrs, or eis thus leading to pre-XDR or XDR phenotype. Most of those mutations were high-confidence, high resistance-level mutations according to the WHO guidelines ${ }^{16}$.

WGS/NGS is becoming less expensive and more available and while NGS technologies are not always accessible especially in remote areas, this could be remedied by centralization of WGS services ${ }^{17}$. However, it is unrealistic to implement NGS for routine TB diagnostics and surveillance in the high burden countries such as Russia given a large size of the country and 50/100,000 incidence i.e., 72,000 new cases in 2019 (https://data.worldbank. org/indicator/SH.TBS.INCD?locations=RU). This highlights the importance and interest in the development of methods for detection of epidemic clusters, ideally less time-consuming than 24-loci VNTR typing but with an equivalent turn-around time and less expensive than WGS.

Here, we identified in silico the SNP markers specific for the Beijing 1071-32-cluster and developed a realtime PCR-based procedure to reliably detect this genotype. We evaluated the specificity of these SNPs on a 


\begin{tabular}{|c|c|c|c|}
\hline Gene, codon & Position in gene & Position in genome & Primer, probe, and sequence \\
\hline Rv0144 74GCC/GCT & $222 \mathrm{C}>\mathrm{T}$ & $170505 \mathrm{C}>\mathrm{T}$ & $\begin{array}{l}\text { 170505F 5'-CCAACGGTAGGTACCAAGC } \\
\text { 170505R 5'-GCTTCCGAGTCTCATCTGCT } \\
\text { 170505C wt 5'-[HEX]GTTCAATGTCGCTCACGGC[C-LNA] } \\
\text { G[BHQ1] } \\
\text { 170505 T mut 5'-[FAM] GTTCAATGTCGCTCACGGC[T-LNA] } \\
\text { G[BHQ1] }\end{array}$ \\
\hline Rv0373c 98CCG/CCA & $294 \mathrm{G}>\mathrm{A}$ & $451510 \mathrm{C}>\mathrm{T}$ & $\begin{array}{l}\text { 451510F 5'-CGCATCGATGTGACTGCC } \\
\text { 451510R 5'-CACGGCTTGTACGTCGTTG } \\
\text { 451510G wt 5'-[HEX]GCCTGGCTTGGATGCC[G-LNA] } \\
\text { ACA[BHQ1] } \\
\text { 451510A mut 5'-[FAM]GCCTGGCTTGGATGCC[A-LNA] } \\
\text { ACA[BHQ1] }\end{array}$ \\
\hline Rv0334 87GCG/GCA & $261 \mathrm{G}>\mathrm{A}$ & $398918 \mathrm{G}>\mathrm{A}$ & $\begin{array}{l}\text { 398918F 5'-GAGTGAACATCAGCTACGC } \\
\text { 398918R 5'-GGCCGTAGAAGATGTTGTC } \\
\text { 398918G wt 5'-[HEX]TCAGCCTGACGGTCTGGC[G-LNA] } \\
\text { CA[BHQ1] } \\
\text { 398918A mut 5'-[FAM]TCAGCCTGACGGTCTGGC[A-LNA] } \\
\text { CA[BHQ1] }\end{array}$ \\
\hline
\end{tabular}

Table 1. Oligonucleotides primers and labeled probes used for detection of three synonymous SNPs specific of the Beijing 1071-32-cluster. wt, wild type; mut, mutant; LNA, locked nucleic acid.

geographically and genetically diverse collection of isolates from East Asia and the former Soviet Union in which the Beijing genotype is endemic or epidemic.

\section{Materials and methods}

Study collections. The study samples included M. tuberculosis DNA collected between 1996 and 2020 either convenience samples or from prospective or cross-sectional studies. Samples were not biased with regard to drug resistance, comorbidities or other patient related characteristics.

The study was approved by the Ethics Committees of the Research Institute of Phthisiopulmonology (protocol 31.2 of February 27, 2017) and St. Petersburg Pasteur Institute (protocol 41 of December 14, 2017).

All methods were performed in accordance with the relevant guidelines and regulations.

Genotyping. DNA was extracted from cultured $M$. tuberculosis isolates using the CTAB-based method ${ }^{18}$ (Russian isolates from northwestern Russia and Omsk, isolates from Belarus, Kazakhstan, Japan, China, Vietnam, and Brazil), DNA-Sorb-B kit (Interlabservis, Russia) (Russian isolates from Irkutsk, Buryatia, Yakutia), «Express-tub» kit (Syntol, Russia) (Russian isolates from Yekaterinburg and Yamalo-Nenets), incubation at $98^{\circ} \mathrm{C}$ and sonication for $15 \mathrm{~min}$ (isolates from Greece), three cycles of hot-freeze cell disruption (isolates from Albania), GenoLyse ${ }^{\infty}$ kit (Hain Lifescience) (isolates from Estonia). One microliter of the DNA extracted using sonication, boiling method or commercial kits and 10-20 ng of DNA extracted using CTAB method was used for PCR.

Spoligotyping and 24 loci MIRU-VNTR typing were performed according to standard protocols ${ }^{19,20}$.

TB Profiler database (http://tbdr.lshtm.ac.uk/) was used for genotypic detection of drug resistance. MDR, pre-XDR and XDR phenotypes were defined according to the World Health Organization definitions: MDR are strains resistant to isoniazid and rifampicin; XDR_-resistant to isoniazid, rifampicin, fluoroquinolone, and a second-line injectable agent; pre-XDR-resistant to isoniazid, rifampicin and either a fluoroquinolone or a second-line injectable agent.

Bioinformatics and phylogenetic analysis. Phylogenetic analysis of the ancient Beijing strains based on the WGS data obtained in our laboratory or retrieved from SRA NCBI, was described previously ${ }^{15}$ and the list of the accession numbers of the analyzed genomes is provided in the same publication. In brief, short sequencing reads were mapped to the reference genome of M. tuberculosis H37Rv (NC_000962.3) by using the Burrows-Wheeler Aligner v. 0.7.12 $2^{21}$. Single nucleotide polymorphism (SNP) calling was performed by SAMtools v.0.1.1922. Variable call format (vcf) files were annotated by BSATool v.0.1 ${ }^{23}$. Graphical presentation of the trees was done using TreeGraph (http://treegraph.bioinfweb.info) and iTOL v3 (http://itol.embl.de). Obtained VCF files were used for analysis. Single nucleotide polymorphisms (SNPs) with quality scores $\geq 20$ were used for phylogenetic analysis with IQTREE software (http://www.cibiv.at/software/iqtree) by using ModelFinder (http:// www.iqtree.org/ModelFinder) with the GTR2 + FO + G4 substitution model and 1,000 rapid bootstrap replicates. SNPs located in repetitive genome regions and in PE and PPE genes were removed from the analysis due to possible misalignments.

To assess the significance of amino acid substitutions, we used 4 parameters: Tang U-index, Grantham D-distance, BLOSUM62 distance, and Dayhoff log odds. They were calculated using in-house scripts, in particular, the snpMiner2 program $^{24}$.

Real-time PCR. Three SNPs at genome positions 170505, 451510 and 398918 specific of the Beijing 1071-32 cluster were tested by real-time PCR assays that were run under the same cycling conditions. Oligonucleotide primers and FAM and HEX labeled probes are listed in Table 1. 


\begin{tabular}{|l|l|l|l|l|}
\hline Gene & Genome position & Method of detection & Primers and RE (in case of PCR-RFLP) & Reference \\
\hline KatG 315 AGC/ACC & 2155168 & PCR-RFLP & $\begin{array}{l}\text { Fw 5'-GCAGATGGGGCTGATCTACG } \\
\text { Rev 5'-AACGGGTCCGGGATGGTG } \\
\text { RE: MspI }\end{array}$ & 26 \\
\hline KatG 335 ATC/GTC & 2155109 & PCR-RFLP & $\begin{array}{l}\text { Fw 5'-GCAGATGGGGCTGATCTACG } \\
\text { Rev 5'-AACGGGTCCGGGATGGTG } \\
\text { RE: AvaII }\end{array}$ & This study \\
\hline RpoB 450 TCG/TTG & 761155 & Rifoligotyping & $\begin{array}{l}\text { Fw 5'-GTCGCCGCGATCAAGGA } \\
\text { Rev 5'-[Biotin]ACGTCGCGGACCTCCA }\end{array}$ & 25 \\
\hline RpoC 485 GAT/AAT & 764822 & Allele-specific PCR & $\begin{array}{l}\text { Fw 5'-CTGGAGCTGTTCAAGCCGTTC } \\
\text { RevWT 5'-CGATGACCTCTTCGAGCACAGC } \\
\text { RevMut 5'-CGATGACCTCTTCGAGCACAGC }\end{array}$ & This study \\
\hline EmbB 497 CAG/CGG & 4248003 & PCR-RFLP & $\begin{array}{l}\text { Fw 5'-TGTGCAGCCCACCGGCCTGA } \\
\text { Rev 5'-TGTGCAGCCCACCGGCCTGA } \\
\text { RE: MspI }\end{array}$ & This study \\
\hline RpsL L43 AAG/AGG & 781687 & PCR-RFLP & $\begin{array}{l}\text { Fw 5'-CAGCCCGCAGCGTCGTGGTG } \\
\text { Rev 5'-GCTGCGTGCCTGTTTGCGGTTCTT } \\
\text { RE: MboII }\end{array}$ & This study \\
\hline
\end{tabular}

Table 2. Molecular assays used for detection of six drug resistance/compensatory mutations specific of the Beijing 1071-32-cluster. RE, restriction endonuclease.

PCR mix $(25 \mu \mathrm{l})$ contained $3 \mathrm{mM} \mathrm{MgCl} 2,1$ unit of hot start Taq DNA polymerase, $200 \mu \mathrm{M}$ each of dNTP, and primers ( 8 pmol each) and probes ( 4 pmol each). RotorGene6000 (Corbette Research) and CFX96 (Biorad) thermal cyclers were used with the following cycling conditions: $95^{\circ} \mathrm{C}, 10 \mathrm{~min}$; followed by 40 cycles of $95^{\circ} \mathrm{C}$, $30 \mathrm{~s} ; 60^{\circ} \mathrm{C}, 10 \mathrm{~s}, 72^{\circ} \mathrm{C}, 20 \mathrm{~s}$ (signal detection at $60^{\circ} \mathrm{C}$ at wavelength $510 \mathrm{~nm}$ for FAM and $555 \mathrm{~nm}$ for HEX). No difference was found between results obtained in different thermal cyclers.

Detection of drug resistance mutations. Six drug resistance mutations were previously shown to be specific for Beijing 1071-32-cluster ${ }^{15}$ : KatG Ser315Thr, KatG Ile335Val, RpoB Ser450Leu, RpoC Asp485Asn, EmbB Gln497Arg, RpsL Lys43Arg. To detect these mutations we used different methods, some of which were previously described, as follows.

Rifoligotyping reverse hybridization assay was used to detect mutations in the $r p o B$ hot-spot region ${ }^{25}$. Other mutations were detected using allele-specific PCR or PCR-RFLP assays followed by $1.5 \%$ agarose gel electrophoresis (Table 2). All methods were initially tested and optimized using DNA samples of Beijing 1071-32 isolates with available WGS data and H37Rv as wild type control.

Allele-specific PCR was used to specifically detect $r p o C 485$ GAT $>$ AAT mutation; primers were selected using WASP online tool at https://bioinfo.biotec.or.th/WASP/home. Both allele-specific primers contained mismatch at -1 position at $3^{\prime}$-end to increase instability during annealing and increase specificity. Two separate PCR reactions were performed for each strain at the same PCR conditions and using the same forward primer and allele-specific mutant primers. PCR products for both reactions are identical $130 \mathrm{bp}$ and are subjected to gel-electrophoresis in adjacent lanes for each strain for easier comparison.

katG315 AGC>ACC mutation was detected using PCR-RFLP assay as described ${ }^{26}$. This mutation creates an additional site for MspI and the main digestion bands have a size of 153 and $132 \mathrm{bp}$ in the case of wild type and mutant alleles, respectively.

katG335 ATC>GTC mutation was detected using PCR-RFLP assay. This mutation creates an additional site for AvaII and the main digestion products have sizes 378 and $285 \mathrm{bp}$ in the case of wild type and mutant alleles, respectively.

rpsL43 AAG >AGG was detected using PCR-RFLP assay. This mutation occurs within the only MboII site in the PCR product in case of the wild type allele. The main MboII digestion products are 210 and 60 bp in case of wild type allele and $270 \mathrm{bp}$ in case of mutation.

embB497 CAG>CGG was detected using PCR-RFLP assay. This mutation creates an additional site for MspI and the main digestion products have sizes 170 and $135 \mathrm{bp}$ in the case of wild type and mutant alleles, respectively.

\section{Results}

Identification of Beijing 1071-32-cluster specific SNPs. Phylogenetic analysis of 184 WGS samples of ancient Beijing sublineages demonstrated that all isolates with Beijing 1071-32 VNTR profile were also grouped within a monophyletic branch on the WGS tree ${ }^{15}$ (Fig. S1). This branch included only Russian isolates (Omsk region in Siberia, St. Petersburg in Northwestern Russia, Samara in Central Russia). For the purposes of the present study, we reanalyzed the genome variation data underlying this tree and identified SNPs that were specific for this branch that we termed "Beijing 1071-32-cluster", i.e. 1071-32 and its single/double locus variants (Fig. S2). In total, 121 SNPs were identified: 15 in intergenic regions and 106 in the coding sequences. These latter included 56 nonsynonymous ( 2 nonsense and 54 missense) and 39 synonymous mutations. In silico based assessment of the significance of the amino acid substitutions revealed three synonymous mutations in $R v 0144, R v 0373 c$, and $R v 0334$ with null significance score by all four tested parameters (Tang U-index, Grantham D-distance, BLOSUM62 distance, and Dayhoff log odds). We used these three SNPs for the development of real-time PCR assays (Table 1). The synonymous SNPs reflect a neutral evolution non-influenced by selection pressure and unlikely to independently occur in different and unrelated phylogenetic groups. Moreover, Rv0334 


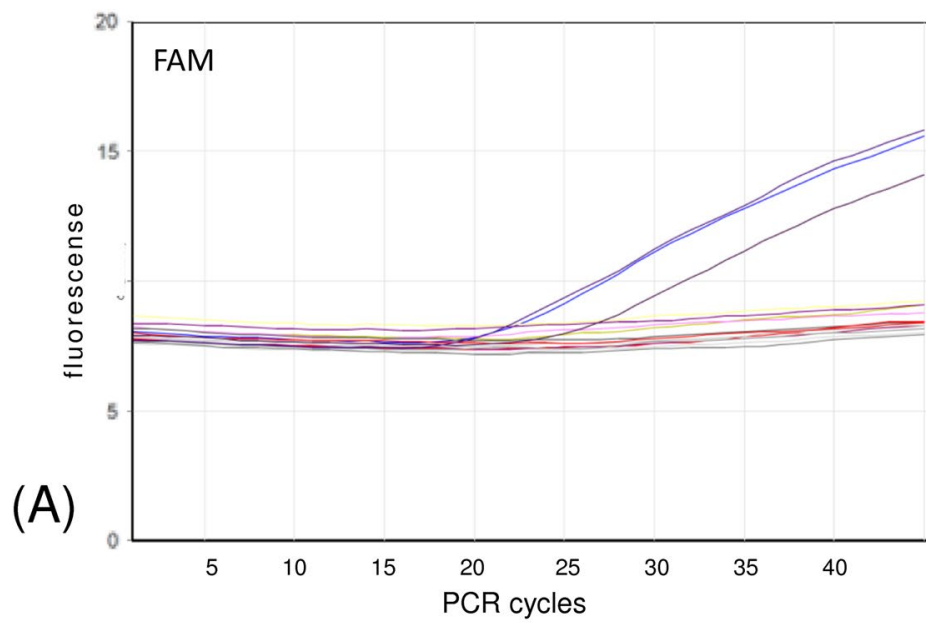

Beijing 1071-32-cluster

Other genotype,

water

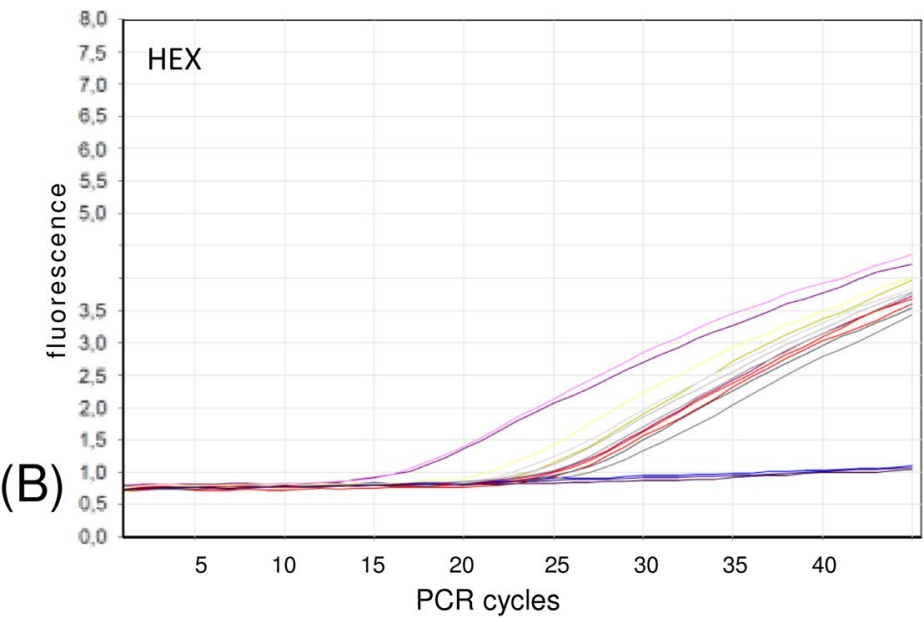

Other genotype

Beijing 1071-32-cluster, water

Figure 2. Fluorescence curves of a real-time PCR assay targeting Rv0144 SNP. (A) Beijing 1071-32 specific signal (FAM channel, $510 \mathrm{~nm}$ ); (B) other genotype-specific signal (HEX channel, $555 \mathrm{~nm}$ ). Water serves as negative control sample.

codes for a thymidylyltransferase from the lipopolysaccharide biosynthetic pathway deemed essential by analysis of saturated Himar1 transposon libraries ${ }^{27}$ and therefore unlikely to be affected by genomic deletion events.

We evaluated in silico the specificity of these SNPs by search in the M. tuberculosis genome diversity GMTV database ${ }^{28}$. They were not detected in the non-Beijing isolates and were detected only in 6 Beijing isolates from Samara, Central Russia included in the above WGS analysis i.e. isolates of this Beijing 1071-32-cluster itself.

Real-time PCR: optimization, validation and screening. Three SNPs were targeted by three separate real-time PCR reactions run simultaneously under the same cycling conditions. Initial optimization was performed with DNA samples with available WGS and VNTR data that represented different VNTR clusters of the Beijing genotype and $\mathrm{H} 37 \mathrm{Rv}$ reference strain. Clear discrimination between wild type and mutant alleles was observed for all three tested SNPs (see an example for one SNP in Fig. 2). The DNA used for analysis was extracted from cultured bacteria using different methods but this did not affect the performance of the assay.

The three RT-PCR assays were further applied to the 675 Beijing genotype isolates that represented different Beijing sublineages and had different VNTR profiles. These validation collections included isolates from Russia $(\mathrm{n}=378)$, Kazakhstan $(\mathrm{n}=109)$, China $(\mathrm{n}=45)$, Vietnam $(\mathrm{n}=37)$, Japan $(\mathrm{n}=71)$, Albania $(\mathrm{n}=5)$, Greece $(\mathrm{n}=19)$, Brazil $(n=11)$. This analysis demonstrated that all 48 Beijing 1071-32-cluster isolates had these three mutations. In contrast, all other 627 isolates with other VNTR profiles had wild type alleles in these genome positions. Thus, this method has $100 \%$ sensitivity and 100\% specificity to detect Beijing 1071-32-cluster.

We further applied these RT-PCR assays to screen all available DNA collections from Russian regions and other countries. Results summarizing the above validation and screening analysis based on the total of 2375 isolates are shown in Table 3 and Fig. 3.

Drug resistance mutational signature in the Beijing 1071-32-cluster. Under the previous WGS analysis, the isolates of the Beijing 1071-32-cluster were shown to have multiple mutations in drug resistance genes and, in particular, were characterized by the specific signature of 6 mutations kat $315 \mathrm{AGC} / \mathrm{ACC}$, kat $\mathrm{G}$ 335ATC/GTC, rpoB 450TCG/TTG, rpoC 485GAT/AAT, embB 497CAG/CGG, rpsL 43AAG/AGG ${ }^{15}$. 


\begin{tabular}{|l|c|l|}
\hline Country, region, years & Beijing & Beijing 1071-32 \\
\hline Russia, Northwest, CП6 1996-2020 & 835 & 22 \\
\hline Russia, Northwest, Karelia, 2014 & 43 & 6 \\
\hline Russia, Northwest, Pskov, 2015, 2018 & 85 & 3 \\
\hline Russia, Northwest, Kaliningrad, 2015 & 46 & 1 \\
\hline Russia, Northwest, Komi, 2017 & 73 & 1 \\
\hline Russia, Northwest, Vologda, 2018 & 51 & 0 \\
\hline Russia, Northwest, Murmansk, 2017 & 32 & 0 \\
\hline Russia. Siberia, Omsk, 2008-2019 & 372 & 37 \\
\hline Russia, Siberia, Irkutsk, 2014-2015 & 147 & 2 \\
\hline Russia, Ural, Yamalo-Nenets, 2017 & 101 & 1 \\
\hline Russia, Ural, Yekaterinburg, 2019-2020 & 85 & 2 \\
\hline Russia, Far East, Yakutia, 2012 & 67 & 1 \\
\hline Albania, 2007-2011 & 5 & 1 \\
\hline Greece, 2008-2011 & 19 & 3 \\
\hline Estonia, 1999 and 2014 & 91 & 0 \\
\hline Belarus, 2004 & 50 & 0 \\
\hline Kazakhstan, 2010 & 109 & 0 \\
\hline China, 2004 & 45 & 0 \\
\hline Vietnam, 2005 & 37 & 0 \\
\hline Japan, 2003-2005 & 71 & 0 \\
\hline Brazil, 2001-2007 & 11 & 0 \\
\hline Total & 2375 & 80 \\
\hline
\end{tabular}

Table 3. Detection of the Beijing 1071-32-cluster by the developed PCR assay in local collections. Beijing genotype was detected using spoligotyping, or PCR targeting dnaA-dnaN::IS6110 (Mokrousov et al., 2014) or based on VNTR-clustering. Beijing 1071-32-cluster was detected by real-time PCR analysis of SNPs in Rv0144, $R v 0373 c$, and $R v 0334$ as described in this study. Geographic locations are shown on Fig. 3.

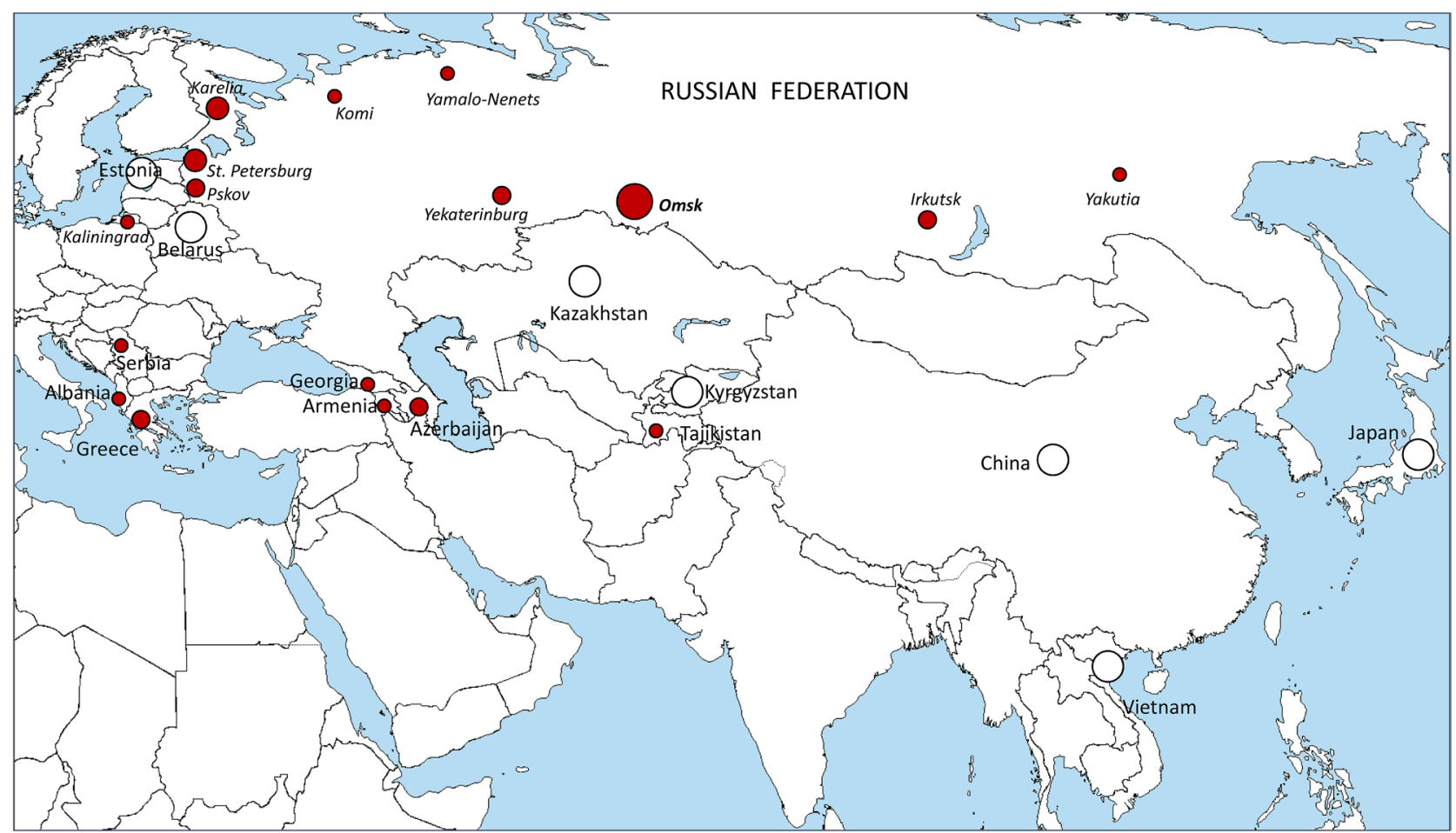

Figure 3. Geographic distribution of the Beijing 1071-32-cluster isolates identified in this study. Circle size roughly correlates with the proportion of identified isolates of this cluster (the smallest dots depict single isolates). Absence of these isolates in the local populations is shown by white circle. No Beijing 1071-312-cluster isolates were detected in Brazil (not shown on the map), Free map: https://commons.wikimedia.org/wiki/ File:A_large_blank_world_map_with_oceans_marked_in_blue.PNG. 
We screened the above-identified Beijing 1071-32-cluster isolates for the presence of these mutations. As a result, all Beijing 1071-32-cluster isolates harbored these six mutations.

\section{Discussion}

This study was undertaken to develop a practical approach to robust detection and prospective surveillance of the MDR/pre-XDR cluster within M. tuberculosis Beijing genotype emerging in Russia ${ }^{14}$. WGS-based analysis of the Russian and global datasets demonstrated that this Beijing 1071-32 cluster also presents a separate branch on the WGS-based tree ${ }^{15}$. In terms of large-scale sublineages, this cluster belongs to the early ancient Beijing sublineage characterized by deleted RD181 and wild type mutT2-58 and mutT4-48 codons (Fig. 1). In terms of drug resistance, it bears a characteristic signature of six drug resistance mutations associated with resistance to the four first-line drugs ${ }^{15}$. Additional mutations in different genes were also present in most isolates of that branch and were associated with resistance to one or more second-line drugs, leading to pre-XDR or XDR genotype (Fig. S2). Most noteworthy and alarmingly, this cluster included only MDR isolates. For example, even the notorious Russian epidemic B0/W148-cluster reportedly included not only MDR but polyresistant isolates resistant to STR and RIF or INH ${ }^{8,29,30}$.

On the whole, based on the VNTR analysis, Beijing 1071-32-cluster isolates were mostly detected in Russia with clearly increasing prevalence in Omsk, Siberia but these isolates were also present in other Russian regions and FSU countries in Central Asia and Transcaucasia (Fig. 3). In the large Beijing genotype VNTR dataset ${ }^{31}$, this VNTR profile 1071-32 was identified in the isolates from Armenia (1 isolate) and Abkhazia (5 isolates). When we additionally searched for the three neutral SNPs and six resistance mutations in the large Beijing genotype WGS dataset previously compiled and described in Perdigão et al. ${ }^{13}, 5$ more isolates that harbored these mutations were detected. They originated from the FSU countries Azerbaijan $(n=3)$, Georgia $(n=1)$ and Tajikistan $(n=1)$.

Outside FSU, the Beijing 1071-32 isolates were described in Serbia ${ }^{31}$, Albania ${ }^{32}$ and Greece ${ }^{33}$. These are Balkan countries but whether this fact reflects an already local circulation of this strain rather than independent importations from FSU is presently unknown. DNA from Albania and Greece were available for this study and we confirmed that they harbored all 9 targeted mutations i.e., 3 neutral SNPs and 6 resistance mutations. The three isolates from Greece were one from an immigrant from Georgia (FSU) and the remainder two from local Greek patients ${ }^{33}$. Moreover, Beijing genotype is extremely rare in Albania ${ }^{32}$ and it was previously speculated that these isolates in Albania could be linked to neighboring Greece. A large European multicenter study of drug resistant TB in the EU ten years ago identified the Beijing 1071-32 isolates and designated them as European resistant cluster ECDC_10 $10^{34}$. This additionally highlights the presence of Beijing 1071-32 MDR strains in the $\mathrm{EU}$ and, given its association with MDR-TB, the relevance of their tracing on a more global scale not limited to Russia and other FSU countries.

Re-analysis of the different typing data available in our laboratory revealed that VNTR 1071-32 profile was detected in Russian strains isolated in 1996-1999 and these strains had a characteristic IS6110-RFLP profile designated as P0 in the in-house database ${ }^{29}$ (Fig. S3). Although the former gold standard IS6110-RFLP typing is almost never used anymore, this knowledge may be helpful to trace these isolates in the historical collections with IS6110-RFLP profiles available, e.g. RIVM database (Bilthoven, The Netherlands) or PHRI database (New Jersey, USA), thereby providing backward compatibility with more modern typing methods and WGS. We have also noticed that all Beijing 1071-32 isolates had 1 copy in the MIRU10 locus. This marker may serve for preliminary detection of these isolates among those assigned to the ancient sublineage of the Beijing genotype.

A strategy to target a limited number of SNPs (at least two) was recommended and applied to identify specific strains or clones by PCR based assays ${ }^{11,35-37}$ which definitely increases the robustness of identification. Thus, application of the entire set of the three targeted SNPs can be seen as the most robust method to detect the Beijing 1071-32-cluster. Nonetheless, detection of particular clusters/genotypes based on use of a single marker is an acceptable and parsimonious approach, provided that such marker was proven specific and sensitive in the validation studies and this concerns both detection of the particular clusters and families and the development of the SNP-barcode system ${ }^{6,9,38,39}$. In this view, since analysis of the three SNPs showed completely concordant results, testing of any of them appears the most practical and time-saving approach to trace this clinically significant MDR Beijing 1071-32-cluster.

Enigmatically, no fully susceptible or mono/polyresistant strains of this MDR cluster were identified yet, and circumstances of its origin remain unknown. Given that only 13 isolates with NGS data from three locations in Russia were analyzed in our previous paper ${ }^{15}$, we could not rule out that isolates with partial resistance profile could be identified in the large and geographically diverse collection in this study. However, no such isolates were detected and the fact that all geographically diverse isolates of this cluster bear the same 6-mutation drug resistance signature is remarkable. This variant included only MDR isolates and this finding favors the hypothesis of dissemination exclusively driven by primary resistance, rather than independent acquisition of resistances.

A Russian origin of this cluster is the most plausible scenario since it includes isolates from remote parts of Russia and some of the analyzed local collections in this study date back to 1996 (St. Petersburg). All identified strains of Beijing 1071-32, regardless of their origin, had all 6 resistance mutations. Given the geographic diversity of the isolates, this probably reflects the distant time of origin of this resistance mutation signature. Phylogenomic analysis suggested an origin of this Russian resistant cluster in the $1970 \mathrm{~s}^{15}$ when RIF was first included in anti-TB chemotherapy ${ }^{40}$. This scenario finds parallel situations across Portugal and South Africa where M/XDR-TB endemic strains of the LAM lineage (e.g. Lisboa3 or KZN, respectively) are predicted to evolve to MDR following the introduction of RIF ${ }^{41,42}$.

The assay showed a good performance with diluted DNA extracted using simplified boiling procedure and we believe it may also work with DNA extracted from clinical samples using commercial kits. Such rapid detection 
would be especially useful also to reliably predict the MDR pattern since all isolates of this cluster were shown to be quadruple resistant.

In conclusion, this study provides a set of three concordant SNPs for the detection and screening of Beijing 1071-32 isolates along with a validated real-time PCR assay easily deployable across multiple settings for the epidemiological tracking of this important MDR cluster. Application of the entire set of the three targeted SNPs can be seen as the most robust method to detect the Beijing 1071-32-cluster. However, since analysis of these three SNPs showed concordant results, testing of any of them appears the practical and time-saving approach to trace this clinically significant emerging cluster. This assay may be especially useful in high MDR-TB burden and/or resource-limited countries of the former Soviet Union and in many world regions that receive migration flows from the FSU countries.

\section{Data availability}

The data that support the findings of this study are available from the corresponding author, I.M., upon request.

Received: 30 July 2021; Accepted: 19 October 2021

Published online: 01 November 2021

\section{References}

1. Coscolla, M. \& Gagneux, S. Consequences of genomic diversity in Mycobacterium tuberculosis. Semin. Immunol. 26, 431-444 (2014).

2. Mokrousov, I. et al. Phylogenetic reconstruction within Mycobacterium tuberculosis Beijing genotype in northwestern Russia. Res. Microbiol. 153, 629-637 (2002).

3. Ebrahimi-Rad, M. et al. Mutations in putative mutator genes of Mycobacterium tuberculosis strains of the W-Beijing family. Emerg. Infect. Dis. 9, 838-845 (2003).

4. Kremer, K. et al. Definition of the Beijing/W lineage of Mycobacterium tuberculosis on the basis of genetic markers. J. Clin. Microbiol. 42, 4040-4049 (2004).

5. Bifani, P. J., Mathema, B., Kurepina, N. E. \& Kreiswirth, B. N. Global dissemination of the Mycobacterium tuberculosis W-Beijing family strains. Trends Microbiol. 10, 45-52 (2002).

6. Millán-Lou, M. I. et al. Rapid test for identification of a highly transmissible Mycobacterium tuberculosis Beijing strain of subSaharan origin. J. Clin. Microbiol. 50, 516-518 (2012).

7. Schürch, A. C. et al. Mutations in the regulatory network underlie the recent clonal expansion of a dominant subclone of the Mycobacterium tuberculosis Beijing genotype. Infect. Genet. Evol. 11, 587-597 (2011).

8. Mokrousov, I. Insights into the origin, emergence, and current spread of a successful Russian clone of Mycobacterium tuberculosis. Clin. Microbiol. Rev. 26, 342-360 (2013).

9. Shitikov, E. et al. Simple assay for detection of the central Asia outbreak clade of the Mycobacterium tuberculosis Beijing genotype. J. Clin. Microbiol. 57, e00215-e219 (2019).

10. Hur, Y. G. et al. Host immune responses to antigens derived from a predominant strain of Mycobacterium tuberculosis. J. Infect. 73, 54-62 (2016).

11. Pérez-Lago, L. et al. Urgent implementation in a hospital setting of a strategy to rule out secondary cases caused by imported extensively drug-resistant Mycobacterium tuberculosis strains at diagnosis. J. Clin. Microbiol. 54, 2969-2974 (2016).

12. Ghebremichael, S. et al. Drug resistant Mycobacterium tuberculosis of the Beijing genotype does not spread in Sweden. PLoS ONE 5, e10893. https://doi.org/10.1371/journal.pone.0010893 (2010).

13. Perdigão, J. et al. Emergence of multidrug-resistant Mycobacterium tuberculosis of the Beijing lineage in Portugal and GuineaBissau: a snapshot of moving clones by whole-genome sequencing. Emerg. Microbes. Infect. 9, 1342-1353 (2020).

14. Mokrousov, I. et al. Early ancient sublineages of Mycobacterium tuberculosis Beijing genotype: unexpected clues from phylogenomics of the pathogen and human history. Clin. Microbiol. Infect. 25(1039), e1-1039.e6 (2019).

15. Mokrousov, I. et al. Genomic signatures of drug resistance in highly resistant Mycobacterium tuberculosis strains of the early ancient sublineage of Beijing genotype in Russia. Int. J. Antimicrob. Agents. 56, 106036 (2020).

16. World Health Organisation. The Use of Next-Generation Sequencing Technologies for Detection of Mutations Associated with Drug Resistance in Mycobacterium tuberculosis Complex: Technical Guide (World Health Organisation, 2018).

17. Nikolayevskyy, V. et al. Role and value of whole genome sequencing in studying tuberculosis transmission. Clin. Microbiol. Infect. 25, 1377-1382 (2019).

18. van Embden, J. D. A. et al. Strain identification of Mycobacterium tuberculosis by DNA fingerprinting: Recommendations for a standardized methodology. J. Clin. Microbiol. 31, 406-409 (1993).

19. Mokrousov, I. \& Rastogi, N. Spacer-based macroarrays for CRISPR genotyping. Methods Mol. Biol. 1311, 111-131 (2015).

20. Supply, P. et al. Proposal for standardization of optimized mycobacterial interspersed repetitive unit-variable-number tandem repeat typing of Mycobacterium tuberculosis. J. Clin. Microbiol. 44, 4498-4510 (2006).

21. Li, H. \& Durbin, R. Fast and accurate short read alignment with burrows-wheeler transform. Bioinformatics 25, 1754-1760 (2009).

22. Li, H. et al. The sequence alignment/map (SAM) format and SAMtools. Bioinformatics 25, 2078-2079 (2009).

23. Sinkov, V. vsink/bsatool: First beta pre-release (Version 0.1). https://doi.org/10.5281/zenodo.3352204 (2019)

24. Sinkov, V., snpMiner2: vcf files annotation tool. https://github.com/vsink/snpminer2 (2016)

25. Morcillo, N. et al. A low cost, home-made, reverse-line blot hybridisation assay for rapid detection of rifampicin resistance in Mycobacterium tuberculosis. Int. J. Tuberc. Lung Dis. 6, 959-965 (2002).

26. Mokrousov, I. et al. High prevalence of KatG Ser315Thr substitution among isoniazid-resistant Mycobacterium tuberculosis clinical isolates from northwestern Russia, 1996 to 2001. Antimicrob. Agents Chemother. 46, 1417-1424 (2002).

27. DeJesus, M. A. et al. Comprehensive essentiality analysis of the Mycobacterium tuberculosis genome via saturating transposon mutagenesis. MBio 8, e0213316. https://doi.org/10.1128/mBio.02133-16 (2017).

28. Chernyaeva, E. N. et al. Genome-wide Mycobacterium tuberculosis variation (GMTV) database: A new tool for integrating sequence variations and epidemiology. BMC Genomics 15, 308. https://doi.org/10.1186/1471-2164-15-308 (2014).

29. Narvskaya, O., Mokrousov, I., Otten, T. \& Vishnevsky, B. Molecular markers: application for studies of Mycobacterium tuberculosis population in Russia. In Trends in DNA Fingerprinting Research (ed. InL Read, M. M.) 111-125 (Nova Science Publishers, 2005).

30. Vyazovaya, A. et al. Increased transmissibility of Russian successful strain Beijing B0/W148 of Mycobacterium tuberculosis: Indirect clues from history and demographics. Tuberculosis (Edinb) 122, 101937 (2020).

31. Merker, M. et al. Evolutionary history and global spread of the Mycobacterium tuberculosis Beijing lineage. Nat. Genet. 47, 242-249 (2015).

32. Tafaj, S. et al. Peculiar features of the Mycobacterium tuberculosis population structure in Albania. Infect. Genet. Evol. 78, 104136 (2020). 
33. Ioannidis, P. et al. Multidrug-resistant/extensively drug-resistant tuberculosis in Greece: predominance of Mycobacterium tuberculosis genotypes endemic in the Former Soviet Union countries. Clin. Microbiol. Infect. 23, 1002-1004 (2017).

34. De Beer, J. L. et al. Molecular surveillance of multi- and extensively drug-resistant tuberculosis transmission in the European Union from 2003 to 2011. Euro Surveill. 19, 20742 (2014).

35. Stucki, D. et al. Tracking a tuberculosis outbreak over 21 years: strain-specific single-nucleotide polymorphism typing combined with targeted whole-genome sequencing. J. Infect. Dis. 211, 1306-1316 (2015).

36. Genestet, C. et al. Expanded tracking of a Beijing Mycobacterium tuberculosis strain involved in an outbreak in France. Travel Med. Infect. Dis. 17, 102167 (2021)

37. Martínez-Lirola, M. et al. Integrative transnational analysis to dissect tuberculosis transmission events along the migratory route from Africa to Europe. J. Travel Med. 28, taab054 (2021).

38. Homolka, S. et al. High resolution discrimination of clinical Mycobacterium tuberculosis complex strains based on single nucleotide polymorphisms. PLoS ONE 7, e39855. https://doi.org/10.1371/journal.pone.0039855 (2012).

39. Napier, G. et al. Robust barcoding and identification of Mycobacterium tuberculosis lineages for epidemiological and clinical studies. Genome Med. 12, 114. https://doi.org/10.1186/s13073-020-00817-3 (2020).

40. Iseman, M. D. Tuberculosis therapy: Past, present and future. Eur. Respir. 36, 87s-94s (2002).

41. Perdigão, J. et al. Using genomics to understand the origin and dispersion of multidrug and extensively drug resistant tuberculosis in Portugal. Sci. Rep. 10, 2600 (2020).

42. Cohen, K. A. et al. Evolution of extensively drug-resistant tuberculosis over four decades: Whole genome sequencing and dating analysis of Mycobacterium tuberculosis isolates from KwaZulu-Natal. PLoS Med. 12, e1001880. https://doi.org/10.1371/journal. pmed.1001880 (2015).

\section{Acknowledgements}

This study was supported by Russian Science Foundation (Grant 19-14-00013).

\section{Author contributions}

I.M. designed the experiment, conducted data analysis and wrote the main manuscript text. A.V., A.G., S.Z., T.U., S.T., P.S. conducted genotyping. V.S., O.O., P.K., J.P. conducted genome analysis and oligonucleotide probe selection. P.I., W.W.J., D.P., O.P., N.R., A.S., Y.S., N.S., N.V., I.Y., D.V., V.Z. cultivated mycobacteria and performed drug susceptibility testing.

\section{Competing interests}

The authors declare no competing interests.

\section{Additional information}

Supplementary Information The online version contains supplementary material available at https://doi.org/ 10.1038/s41598-021-00890-7.

Correspondence and requests for materials should be addressed to I.M.

Reprints and permissions information is available at www.nature.com/reprints.

Publisher's note Springer Nature remains neutral with regard to jurisdictional claims in published maps and institutional affiliations.

(c) (i) Open Access This article is licensed under a Creative Commons Attribution 4.0 International License, which permits use, sharing, adaptation, distribution and reproduction in any medium or format, as long as you give appropriate credit to the original author(s) and the source, provide a link to the Creative Commons licence, and indicate if changes were made. The images or other third party material in this article are included in the article's Creative Commons licence, unless indicated otherwise in a credit line to the material. If material is not included in the article's Creative Commons licence and your intended use is not permitted by statutory regulation or exceeds the permitted use, you will need to obtain permission directly from the copyright holder. To view a copy of this licence, visit http://creativecommons.org/licenses/by/4.0/.

(C) The Author(s) 2021 